\title{
Local predictability in a simple model of atmospheric balance
}

\author{
G. Gyarmati ${ }^{1}$, I. Szunyogh ${ }^{2}$, and D. J. Patil ${ }^{2}$ \\ ${ }^{1}$ Department of Meteorology, Eötvös Loránd University, Budapest, Hungary \\ ${ }^{2}$ Institute for Physical Science and Technology and Department of Meteorology, University of Maryland, College Park, \\ Maryland, USA
}

Received: 11 September 2001 - Revised: 15 May 2002 - Accepted: 17 May 2002

\begin{abstract}
The 2 degree-of-freedom elastic pendulum equations can be considered as the lowest order analogue of interacting low-frequency (slow) Rossby-Haurwitz and highfrequency (fast) gravity waves in the atmosphere. The strength of the coupling between the low and the high frequency waves is controlled by a single coupling parameter, $\varepsilon$, defined by the ratio of the fast and slow characteristic time scales.

In this paper, efficient, high accuracy, and symplectic structure preserving numerical solutions are designed for the elastic pendulum equation in order to study the role balanced dynamics play in local predictability. To quantify changes in the local predictability, two measures are considered: the local Lyapunov number and the leading singular value of the tangent linear map.

It is shown, both based on theoretical considerations and numerical experiments, that there exist regions of the phase space where the local Lyapunov number indicates exceptionally high predictability, while the dominant singular value indicates exceptionally low predictability. It is also demonstrated that the local Lyapunov number has a tendency to choose instabilities associated with balanced motions, while the dominant singular value favors instabilities related to highly unbalanced motions. The implications of these findings for atmospheric dynamics are also discussed.
\end{abstract}

\section{Introduction}

Although it is widely accepted that predictability varies with the atmospheric flow, the issue of finding a single best measure to quantify this variation has not yet been settled. Measures of local predictability in phase space have been studied and compared for a wide variety of models with atmospheric relevance ranging from systems with few degrees of freedom (e.g. Farrell, 1988, 1990; Trevisan and Legnani, 1995; Lorenz, 1996; Legras and Vautard, 1996; Smith, 1997; Fred-

Correspondence to: I. Szunyogh (szunyogh@glue.umd.edu) eriksen, 2000; Trevisan et al., 2001; Ziehmann et al., 2000) to more realistic atmospheric models with higher complexity (e.g. Lacarra and Talagrand, 1988; Houtekamer, 1991; Molteni and Palmer, 1993; Vukicevic, 1993; Buizza and Palmer, 1995; Ehrendorfer and Errico, 1995; Vannitsem and Nicolis, 1997; Szunyogh et al., 1997; Patil et al., 2001). The aim of the present paper is to further investigate this subject for a low-order, non-dissipative analogue to the atmospheric governing equations that can maintain motions of distinctly different time scales and can accommodate an analogue to balanced atmospheric motions.

The first low-order model to study interactions between motions of different time scales in the atmosphere was derived by finding the lowest order truncation of the shallow water equations (Lorenz, 1986). This model, today known as the five-variable Lorenz (L5) model, is a nonlinearly coupled system of two simple integrable mechanical subsystems: a nonlinear pendulum and a harmonic oscillator $(\mathrm{Ca}-$ massa, 1995; Bokhove and Shepherd, 1996). For appropriate choices of the control parameters the frequency of the rotation of the pendulum is much slower than the frequency of the oscillator. In this case, the "slow" swinging of the pendulum and the "fast" resonance of the oscillator can be considered as analogues to the low frequency Rossby-Haurwitz waves and the high frequency gravity waves in the atmosphere. In a perfectly balanced state, the coordinates (variables) associated with "fast" motions are slaved to the coordinates related to "slow" motions. That is, the "fast" components can be determined from the "slow" components at any given time instance by solving an algebraic balance equation.

Lynch $(1996,2002)$ pointed out that a slight modification of the coupling term in the L5 model leads to the equation of motion for the elastic pendulum, where a mass is suspended from an elastic rod which can rotate and stretch, but can not bend. The role balanced dynamics plays in predictability can, therefore, be addressed by studying either the L5 model or the structurally equivalent elastic pendulum. In this paper, for the sake of physical transparency, we study the elastic pendulum equations. 
To quantify changes in the local predictability of the elastic pendulum we consider two different measures: the leading singular value of the tangent linear map and the local Lyapunov number. These two measures (and/or some of their derivatives) have already been compared in a series of papers (e.g. Trevisan and Legnani, 1995; Szunyogh et al., 1997; Reynolds and Errico, 1999; Samelson, 2001; Ziehmann et al., 2000). The main motivations for revisiting the issue for the elastic pendulum are twofold: (1) Since the elastic pendulum has Hamiltonian structure, its singular value spectrum is symmetric. This symmetry can be exploited to study the relationship between singular values and the local Lyapunov number in a more transparent manner than in a dissipative system. (2) Studies with complex models based on the atmospheric primitive equations (Szunyogh et al., 1997; Thorpe, 1996; Errico, 2000; Montani and Thorpe, 2002) found that for a common choice of the coordinates, unbalanced motions played an important role in local predictability when measured by the dominant singular value. In addition, Szunyogh et al. (1997) demonstrated that the local Lyapunov number measured instabilities associated with nearly balanced dynamics. That paper also conjectured that the difference between the two measures of local predictability and their relationship to the balanced dynamics may be due to the fact that the local Lyapunov number measures predictability for trajectories on the attractor of the system, while the dominant singular value can potentially be associated with trajectories which are initially off the attractor (e.g. Anderson, 1995; Legras and Vautard, 1996; Trevisan and Pancotti, 1998). In this paper, we will demonstrate that the relationship between the predictability measures and the role of balanced dynamics is no different for the elastic pendulum than for dissipative atmospheric models. This suggests, that in terms of balanced dynamics, it is not the existence of an attractor, but the presence of multiple time scales that plays the key role in the differences between the predictability measures. (The elastic pendulum has multiple time scales but no attractor since it is a non-dissipative system).

The outline of the paper is as follows. Section 2 is a brief overview of the measures of local predictability considered here. Section 3 presents the basic dynamical equations and gives a short description of the geometry of the phase space. A new family of symplectic structure preserving numerical solutions for the elastic pendulum is presented in Sect. 4 (technical details are provided in the Appendix), while the main results of the paper on local predictability are presented in Sect. 5. Section 6 offers some conclusions.

\section{Measures of local predictability}

\subsection{The tangent-linear map}

Equations that typically arise in atmospheric dynamics (and in numerical weather prediction), after the partial differential equations that govern the atmospheric motions have been dis- cretized in space (e.g. Kádár et al., 1998), can be written as a system of ordinary differential equations,

$\frac{d \boldsymbol{x}}{d t}=\mathbf{X}(\boldsymbol{x}(t)), \quad \boldsymbol{x}(0)=\boldsymbol{x}_{0}$,

where the state vector, $\boldsymbol{x}(t)$, and the vector field, $\mathbf{X}(\boldsymbol{x}(t))$, have $n$ components.

The time evolution of an infinitesimal perturbation, $\delta \boldsymbol{x}_{\tau}$, to the nonlinear trajectory at $\boldsymbol{x}(t-\tau)$ is determined by the associated tangent-linear equation,

$\frac{d \delta \boldsymbol{x}}{d t}=D \mathbf{X}(\boldsymbol{x}(t)) \delta \boldsymbol{x}, \quad \delta \boldsymbol{x}(t-\tau)=\delta \boldsymbol{x}_{\tau}$,

where $D \mathbf{X}$ is the Jacobian matrix for the vector-valued function $\mathbf{X}(\boldsymbol{x}(t))$ (e.g. Ott, 1993). The solution of the tangentlinear equation defines the tangent-linear map $\mathbf{L}(\boldsymbol{x}(t), \tau)$, for which

$\delta \boldsymbol{x}(t)=\mathbf{L}(\boldsymbol{x}(t), \tau) \delta \boldsymbol{x}_{\tau}$.

Measures that quantify the local predictability at $\boldsymbol{x}(t)$ are traditionally defined by the expansion rate $\|\delta \boldsymbol{x}(t)\| /\left\|\delta \boldsymbol{x}_{\tau}\right\|$ of a properly selected initial perturbation vector $\delta \boldsymbol{x}_{\tau}$, where $\|\cdot\|$ denotes the Euclidean norm. The difference between the local predictability measures (in this paper the local Lyapunov number and the dominant singular value) is in the selection of the initial perturbation vector $\delta \boldsymbol{x}_{\tau}$.

\subsection{Dominant singular value}

Singular Value Decomposition (SVD) (e.g. p. 70-72 Golub and Van Loan, 1983) guarantees that for $\mathbf{L}(\boldsymbol{x}(t), \tau)$ there exist two sets of orthonormal basis vectors, $\left[\boldsymbol{v}_{1}, \ldots, \boldsymbol{v}_{n}\right]$ and $\left[\boldsymbol{u}_{1}, \ldots, \boldsymbol{u}_{n}\right]$, and a set of real, non-negative scalars, $\sigma_{1}, \ldots, \sigma_{n}$ $\left(\sigma_{1} \geq \sigma_{2} \geq \ldots \geq \sigma_{n}\right)$, such that

$\mathbf{L}(\boldsymbol{x}(t), \tau) \boldsymbol{v}_{i}(\boldsymbol{x}(t), \tau)=\sigma_{i}(\boldsymbol{x}(t), \tau) \boldsymbol{u}_{i}(\boldsymbol{x}(t), \tau)$.

The scalars $\sigma_{1}, \ldots, \sigma_{n}$ are the singular values, while $\left[\boldsymbol{v}_{1}, \ldots, \boldsymbol{v}_{n}\right]$ and $\left[\boldsymbol{u}_{1}, \ldots, \boldsymbol{u}_{n}\right]$ are, respectively, the right (initial) and the left (evolved) singular vectors of the matrix that represents the tangent linear map $\mathbf{L}(\boldsymbol{x}(t), \tau)$.

It is important to emphasize that the SVD of a linear operator is not independent of the choice of the local coordinates (components of $\boldsymbol{x}$ ) $, x_{1}, x_{2}, \ldots, x_{n}$; (or of the choice of the inner product if the coordinates are fixed and the inner product is varied). In the atmospheric sciences, the general practice is to choose the coordinates so that the square of the norm generated by the Euclidean inner product becomes a quantity with an energy dimension (Talagrand, 1981; Buizza et al., 1993).

Since $\left[\boldsymbol{v}_{1}, \ldots, \boldsymbol{v}_{n}\right]$ provide an orthonormal basis, any initial perturbation, $\delta \boldsymbol{x}_{\tau}$, can be written as

$\delta \boldsymbol{x}_{\tau}=\sum_{i=1}^{n}<\boldsymbol{v}_{i}(\boldsymbol{x}, \tau), \delta \boldsymbol{x}(t) \tau>\boldsymbol{v}_{i}(\boldsymbol{x}(t), \tau)$,

where $<\cdot, \cdot\rangle$ denotes the Euclidean inner product, which combined with Eqs. (3) and (4) gives

$\delta \boldsymbol{x}(t)=\sum_{i=1}^{n} \sigma_{i}(\boldsymbol{x}(t), \tau)<\boldsymbol{v}_{i}(\boldsymbol{x}(t), \tau), \delta \boldsymbol{x}_{\tau}>\boldsymbol{u}_{i}(\boldsymbol{x}(t), \tau) .(6)$ 
This means that when the uncertainty in the prediction is measured by the Euclidean norm the largest possible amplification of the uncertainty is defined by the first singular value $\sigma_{1}(\boldsymbol{x}(t), \tau)$. The dominant (first) singular value, $\sigma_{1}(\boldsymbol{x}(t), \tau)$, can, therefore, be considered as a measure of local predictability at $\boldsymbol{x}(t)$. (A closely related predictability measure is the finite time Lyapunov exponent, $\Sigma=$ $\tau^{-1} \ln \left(\sigma_{1}(\boldsymbol{x}(t), \tau)\right)$, defined by Ziehmann et al. (2000) in a slightly different form). The dominant singular value, of course, is dependent not only on the phase space location $\boldsymbol{x}(t)$, but also on the choice of the coordinates, and the time interval, $\tau$.

Finally, it should be noted that in atmospheric sciences the predictability at $\boldsymbol{x}(t)$ is typically measured by the dominant singular value associated with the time interval $[t, t+\tau]$ rather than $[t-\tau, t]$. In this paper, we define the dominant singular value based on the past evolution of the trajectory so the results can be directly compared to the local Lyapunov number. None-the-less, our main conclusions on the role of multiple time scales and balanced dynamics are equally valid for both definitions.

\subsection{Local Lyapunov number}

As $t$ goes to infinity, all typical perturbations, $\delta \boldsymbol{x}(t)$ in Eq. (3), turn into the direction, $\boldsymbol{l}(\boldsymbol{x})$, parallel to the first left singular vector defined for an infinitely long time interval, $\boldsymbol{u}_{1}(\boldsymbol{x}, \infty)$. The vector $\boldsymbol{l}(\boldsymbol{x})$ is usually referred to as Lyapunov vector. It is important to emphasize that, although Eq. (4) involves the SVD (which is dependent on the coordinates), the Lyapunov vector is independent of the choice of the coordinates (Legras and Vautard, 1996; Trevisan and Pancotti, 1998). For a short interval of time, $\tau$, the speed of the separation between trajectories can be characterized by the local Lyapunov number

$\lambda(\boldsymbol{x}, \tau)=\frac{\|\mathbf{L}(\boldsymbol{x}, \tau) \boldsymbol{l}(\boldsymbol{x}(t-\tau))\|}{\|\boldsymbol{l}(\boldsymbol{x}(t-\tau))\|}$.

(A closely related predictability measure is the finite sample Lyapunov exponent, $\Lambda=\tau^{-1} \ln (\lambda(\boldsymbol{x}(t), \tau))$, defined by Ziehmann et al. (2000) in a slightly different form.) The local Lyapunov number is always positive with values of greater than one indicating diverging trajectories, and values of smaller than one indicating trajectories that are converging in the phase space. For any given choice of the coordinates, the upper bound for the local Lyapunov number (defined by the related Euclidean norm in Eq. (7)) is equal to the dominant singular value, $\sigma_{1}(\boldsymbol{x}(t), \tau)$, while the lower bound is equal to the smallest singular value, $\sigma_{n}(\boldsymbol{x}(t), \tau)$. The upper bound is reached when the first right singular vector, $\boldsymbol{v}_{1}(\boldsymbol{x}(t), \tau)$, and the Lyapunov vector, $\boldsymbol{l}(\boldsymbol{x}(t-\tau))$ are parallel, since for the given choice of coordinates the first right singular vector defines the direction of the fastest possible growth with respect to the Euclidean norm.

It is important to notice, that when the coordinates are changed the new Lyapunov vector can be easily computed by a coordinate transformation, but the right singular vector has

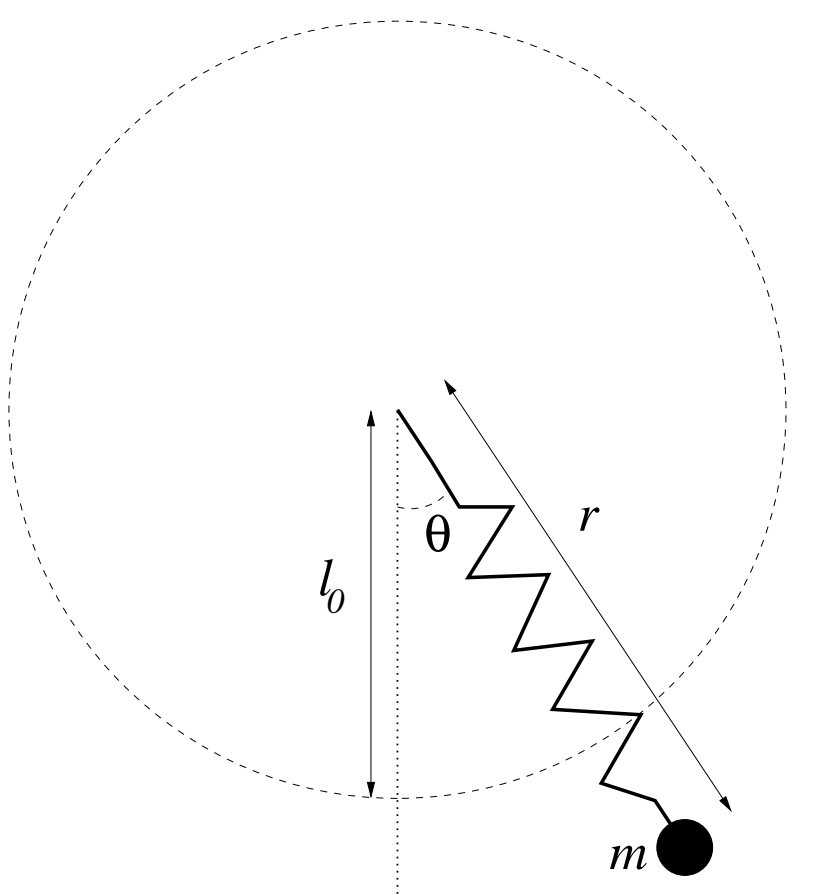

Fig. 1. Schematic picture of the elastic pendulum. The length of the unstretched rod is $l_{0}$, the distance between the origin and the point mass, $m$, is $r$, and the angle of the pendulum is $\theta$. The dashed line shows the circle, along which the pendulum would move if the rod was non-elastic.

to be recomputed. This shows, on the one hand, that while the local Lyapunov number measures expansion (or contraction) associated with the same physical process regardless the choice of the coordinates, the dominant singular value can emphasize different physical processes depending on the choice of the coordinates. On the other hand, the dominant singular value is a truly local quantity, depending only on the trajectory connecting $\boldsymbol{x}(t-\tau)$ and $\boldsymbol{x}(t)$, while the local Lyapunov number is also dependent on the entire trajectory leading to $\boldsymbol{x}(t-\tau)$.

\section{The elastic pendulum}

\subsection{Basic equations}

The elastic pendulum is a point mass, $m$, suspended by a weightless elastic rod, with an unstretched length of $l_{0}$ and spring constant $k$, which may stretch but not bend (see Fig. 1).

The angular oscillation frequency of the pendulum in the limit of small displacement is $\omega_{R}=\sqrt{g / l_{0}}$, where $g$ is the gravitational acceleration. When the displacement is not small the frequency of the rotational motion can be significantly different from $\omega_{R}$ (e.g. Tabor, 1989). The elastic frequency is $\omega_{E}=\sqrt{k / m}$, and the coupling parameter $\varepsilon$ is defined by the ratio $\varepsilon=\omega_{R} / \omega_{E}$. In this paper, only values $\varepsilon \leq 0.4$ are considered. 
For this simple physical system, the nondimensionalized equations of motions can be written as a system of nonlinear ordinary differential equations (Lynch, 1996, 2002)

$$
\begin{aligned}
\frac{d \vartheta}{d t} & =p_{\vartheta}\left(1+\varepsilon^{1 / 2} \rho\right)^{-2}, \\
\frac{d p_{\vartheta}}{d t} & =-\left(1+\varepsilon^{1 / 2} \rho\right) \sin \vartheta, \\
\frac{d \rho}{d t} & =\varepsilon^{-1} p_{\rho}, \\
\frac{d p_{\rho}}{d t} & =\varepsilon^{1 / 2} p_{\vartheta}^{2}\left(1+\varepsilon^{1 / 2} \rho\right)^{-3}-\varepsilon^{-1} \rho+\varepsilon^{1 / 2} \cos \vartheta .
\end{aligned}
$$

The units of mass, length and time are defined, respectively, by the mass of the bob, $m$; the length of the unstretched rod, $l_{0}$; and the inverse of the rotational frequency, $1 / \omega_{R}$. The dynamical variables are defined in a polar coordinate system and rescaled so that they are all of the same order for the typical values of the initial condition. Namely, $\vartheta$ is the angle between the rod and the vertical $(\vartheta=0$ is the angle at rest) and the angular momentum, $p_{\vartheta}$, is positive for counter clockwise rotation. In addition, it is assumed that the amplitude of the elastic motion is small. More precisely, for the cases considered here the distance between the point mass and the fixed end of the rod is $r=1+\varepsilon^{1 / 2} \rho$ and $\rho$ is of the same order as $\vartheta$ and $p_{\vartheta}$. The scaling $p_{\rho}=\varepsilon^{1 / 2} d r / d t(=\varepsilon d \rho / d t)$ ensures that $p_{\rho}$ is of the same order as the other variables.

Using the generalized coordinate and momentum representation, i.e. introducing $\boldsymbol{q}=(\vartheta, \rho)$ and $\boldsymbol{p}=\left(p_{\vartheta}, p_{\rho}\right)$; and defining the Hamiltonian $H(\boldsymbol{q}, \boldsymbol{p})$ by the total energy of the system

$H=\frac{1}{2} \varepsilon^{-1}\left(p_{\rho}^{2}+\rho^{2}\right)+\frac{1}{2} p_{\vartheta}{ }^{2}(1+\varepsilon \rho)^{-2}-\cos \vartheta\left(1+\varepsilon^{1 / 2} \rho\right)$

the equation of motion (8) can be expressed in symplectic Hamiltonian form

$\frac{d \boldsymbol{x}}{d t}=\mathbf{J} \nabla_{\boldsymbol{x}} H$.

Here, $\boldsymbol{x}=(\boldsymbol{q}, \boldsymbol{p})^{T}$,

$\mathbf{J}=\left(\begin{array}{cc}\mathbf{0} & \mathbf{I} \\ -\mathbf{I} & \mathbf{0}\end{array}\right)$

and the symbols $\mathbf{0}$ and $\mathbf{I}$ stand, respectively, for the zero and the unity matrices.

For the elastic pendulum, as for any Hamiltonian system, there are two independent conservation laws

$$
\frac{d H}{d t}=0 ; \quad \frac{d \omega}{d t}=0 ;
$$

where $\omega=d \boldsymbol{p} \wedge d \boldsymbol{q}$ is a differential two-form and the symbol $\wedge$ stands for the exterior-derivative. (e.g. Arnold, 1989; Weintraub, 1997). The first conservation law is the wellknown conservation of energy, while the second conservation law is the conservation of symplectic structure. These conservation laws provide a symmetric singular value spectrum described in the following subsection.

\subsection{The geometry of the phase space}

Due to the conservation of the symplectic structure, the singular values are in pairs defined by the relation $\sigma_{i}(\boldsymbol{x}(t), \tau)=$ $\sigma_{n-i+1}^{-1}(\boldsymbol{x}(t), \tau)$. This means that phase space locations of the largest potential growth (locations where $\sigma_{1}(\boldsymbol{x}(t), \tau)$ is the largest) are also locations of the largest potential decay $\left(\sigma_{n}(\boldsymbol{x}(t), \tau)=\sigma_{1}^{-1}(\boldsymbol{x}(t), \tau)\right)$. This also implies that there are no phase points, where the largest singular value can be smaller than one. This is in contrast to the behavior observed for dissipative systems (Smith et al., 1999; Ziehmann et al., 2000).

For the elastic pendulum there are two generalized coordinates $\left(\vartheta\right.$ and $\rho$ ) and two generalized momenta ( $p_{\vartheta}$ and $p_{\rho}$ ), which means that Eq. (8) is a 2 degree-of-freedom Hamiltonian system. Thus, the phase space is 4-dimensional $(n=4)$; however, due to the conservation of energy, the trajectories are confined to a 3-dimensional space (energy shell). Moreover, when $\varepsilon$ is small, or more formally when $\varepsilon \leq 10^{-48}$, the Kolmogorov-Arnold-Moser (KAM) theorem ensures that remains a positive measure of trajectories on 2-dimensional invariant tori embedded in the 3-dimensional energy shell (Lynch, 1996, 2002). The exceptions are chaotic trajectories that fill the 3-dimensional space between the invariant tori. The invariant tori define the analogue of the atmospheric slow manifold. As $\varepsilon$ increases an increasing number of trajectories become chaotic and the invariant tori gradually disappear.

Lynch $(1996,2002)$ showed numerically that most invariant tori remain practically intact for such large values of $\varepsilon$ as 0.2. Images of the invariant tori in the Poincaré sections, plotted for the $\left(\vartheta, p_{\vartheta}\right)$ and the $\left(\rho, p_{\rho}\right)$ planes, are continuous (typically closed) curves, while images of the chaotic orbits fill areas between the tori, but in finite time numerical computations appear as sets of disconnected dots (examples are shown in Figs. 4-7).

\section{Numerical solutions}

\subsection{A new family of numerical solutions}

For most initial conditions, the elastic pendulum equation cannot be solved analytically as an explicit integral, hence only approximate numerical solutions can be obtained. The errors introduced by the numerical integration scheme and the errors due to uncertain initial conditions (chaos) are generally considered to be two independent sources of forecast error. It should be noted though, that the non-integrable nature of the equations and the chaotic behavior of the Hamiltonian system they describe are closely related (e.g. Tabor, 1989).

The numerical integration schemes can introduce both local integration errors and structural errors into the solutions. The local integration error, defined as the quantitative accuracy of the solution for a time step, is proportional to the time step and the order of the integration scheme. Decreasing 
(increasing) the time step has the same effect as increasing (decreasing) the order of the scheme. When enhanced precision is needed, usually, increasing the order of the scheme is cheaper than decreasing the time step. The possibilities are not unbounded though since the time step cannot be larger than what is necessary to resolve the fastest possible changes in the modeled system. This principle sets an upper bound on the order of the integrator that can be efficiently used to integrate the model at a given level of accuracy. For the elastic pendulum equation this bound is set by the time scale of the oscillator. Because the time unit is equal to the time scale of the slow motion, it can be expected that the largest sufficient time step would decrease as $\varepsilon$ decreases.

Structural errors of the numerical solutions are distortions in the geometric structure (qualitative behavior) of the system (Sanz-Serna, 1992). Structural errors can be severe even if the integration scheme provides high local accuracy. A theorem by Ge and Marsden (1988) states, that a numerical solution with a constant time step cannot preserve both the symplectic structure and the energy for a non-integrable Hamiltonian system. That is, the numerical solutions of the elastic pendulum will be inevitably burdened by structural errors. For this paper, we choose to search for solutions that preserve the symplectic structure. The motivations to do so are that (i) structure preserving schemes conserve the symmetry $\sigma_{i}(\boldsymbol{x}(t), \tau)=\sigma_{n-i+1}^{-1}(\boldsymbol{x}(t), \tau)$ for the singular values; and (ii) structure preserving schemes are known to be more efficient in conserving the energy than energy preserving schemes in conserving the structure.

The elastic pendulum is a non-separable Hamiltonian system, which means that a decomposition $H(\boldsymbol{q}, \boldsymbol{p})=H_{1}(\boldsymbol{q})+$ $H_{2}(\boldsymbol{p})$ does not exist. Therefore, standard structure preserving Runge-Kutta methods (e.g. Sanz-Serna and Calvo, 1994) cannot be applied. To the best of our knowledge, the only way to preserve the structure of a non-separable Hamiltonian system is to construct a symmetric composition scheme. In the Appendix, a family of new first-, second-, fourth-, sixth-, and eighth-order numerical solutions is constructed for the elastic pendulum based on the general results of McLachlan (1995).

\subsection{Selection of the optimal scheme}

The global structural accuracy of the numerical solution is measured by the rms relative energy error,

$$
R M S=\sqrt{\frac{1}{N} \sum_{i=1}^{N}\left(\frac{\left(\tilde{H}_{i}\right)-\tilde{H}_{0}}{\tilde{H}_{0}}\right)^{2}},
$$

which is the relative root-mean-square distance between the constant energy surface of the real system (known from the initial conditions) and the numerical solution for a long trajectory. Here, $N$ is the number of time steps taken along the trajectory, $\tilde{H}_{i}$ is the energy in the numerical solution after the $i$ th time step, and $\tilde{H}_{0}=H(\boldsymbol{q}(0), \boldsymbol{p}(0))$.

In order to compare the efficiency of the different integrators, a work is defined to be the estimated number of function
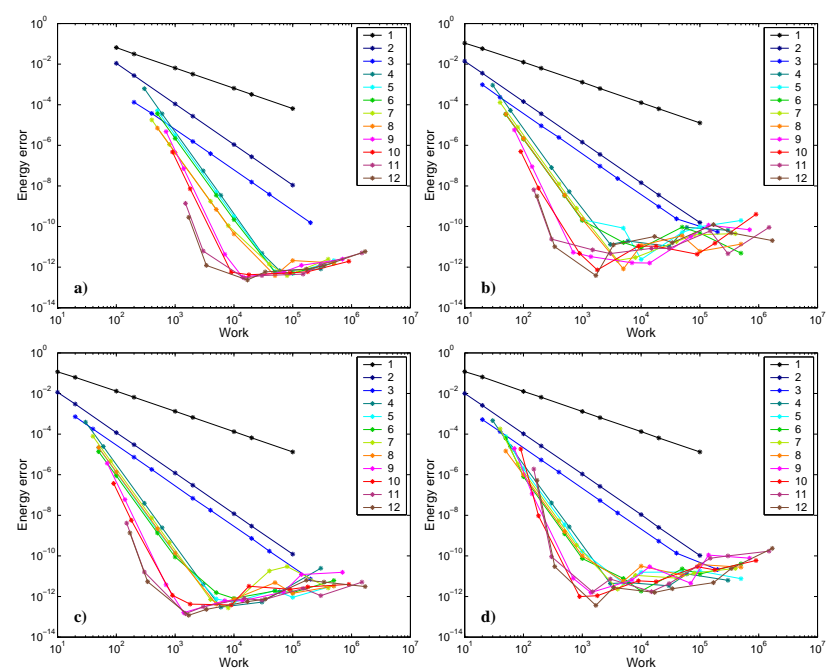

Fig. 2. The rms energy error as function of work (see Sect. 4.2) for the different integration schemes listed in Table A1. The values of the coupling parameter, $\varepsilon$, are 0.025 (panel a), 0.25 (panel b), 0.325 (panel c), and 0.4 (panel d).

evaluations needed to integrate the model for a unit time interval. Then one typical initial condition is selected for each of the four different values of the coupling parameter, $\varepsilon$, considered later, and the numerical solutions are computed for a 2000 time unit interval by each integration scheme listed in Table A1. These computations, as any others reported in this paper, are carried out at 8-byte arithmetic precision.

The rms relative energy error is plotted as function of work for each numerical solution in Fig. 2, where it can be seen that different schemes can be optimal depending on the required accuracy of the solution. In general, the lower order integration schemes are more efficient in producing lower accuracy solutions, while the higher order methods are more efficient when high precision is required.

Since the main concern of this paper is the local predictability of the modelled physical system, it is desirable to increase the local accuracy of the solutions as much as possible. Hence, all predictability experiments are carried out by scheme 12 , which is an eighth order method. For a 0.01 time unit step, which is used in all computations reported hereafter, this scheme provides solutions with an error not larger than $10^{-10}$.

\section{Predictability experiments}

\subsection{Local predictability}

As mentioned before, both local predictability measures investigated here (the dominant singular value and the local Lyapunov number) are dependent on the choice of the time interval $\tau$. Ziehmann et al. (2000) demonstrated by examples that the finite time Lyapunov exponent (a derivative of the dominant singular value) and the finite sample Lyapunov ex- 

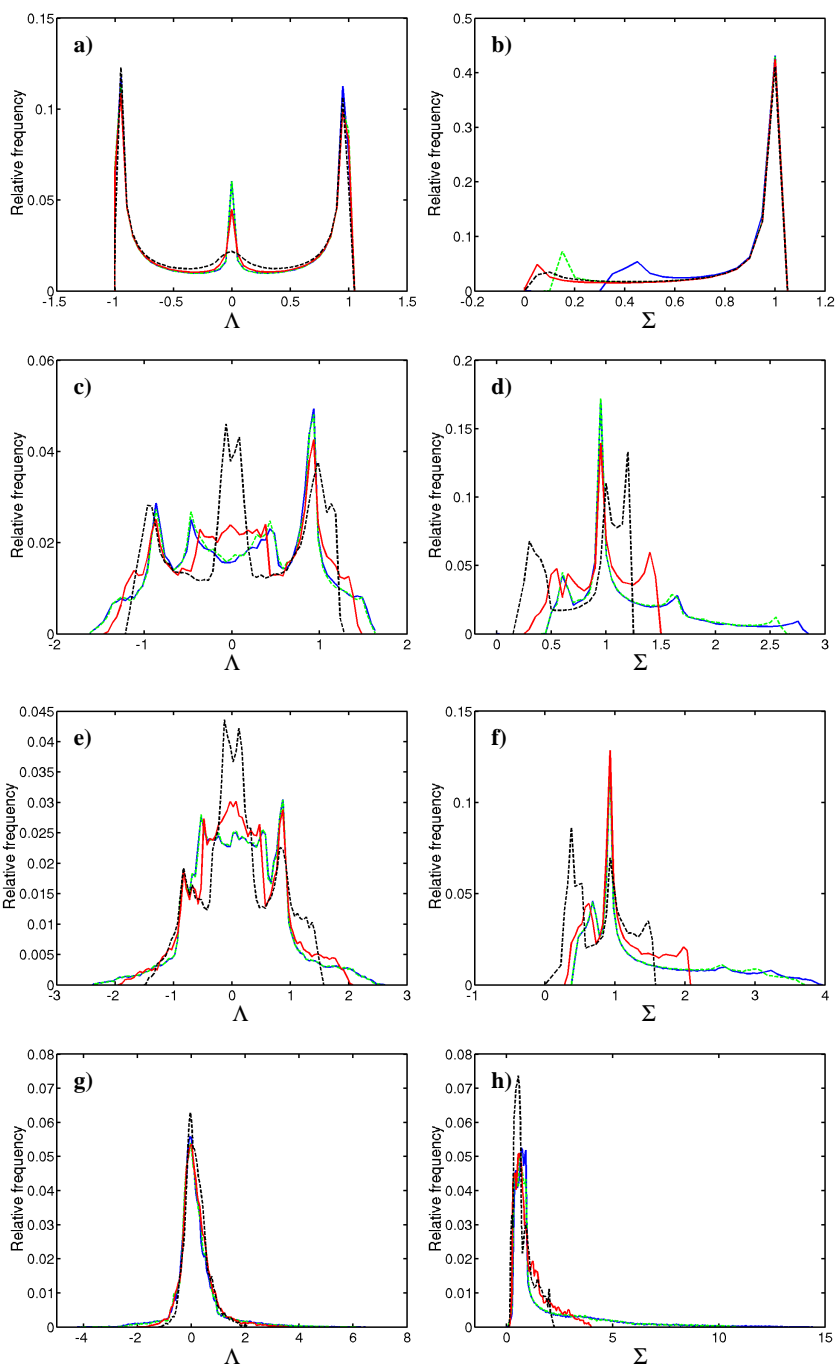

Fig. 3. Each left hand side panel shows the distribution of $\Lambda$, while each right hand side panel shows the distribution of $\Sigma$ for a selected trajectory near the separatrix of the pendulum. The samples were collected for trajectories integrated for 1000 time units. The coupling parameters, $\varepsilon$, are 0.025 (panels a and b), 0.25 (panels $\mathrm{c}$ and d), 0.325 (panels e and f), and 0.4 (panels g and h). The blue, green, red, and black lines show, respectively, the distributions for $\tau=0.01, \tau=0.1, \tau=0.5$, and $\tau=1$.

ponent (a derivative of the local Lyapunov number) are both strongly dependent on the selection of the short time interval. Therefore, in computing the predictability measure, our first task is to select an appropriate $\tau$. In order to make our results comparable to those in the Fig. 1 of Ziehmann et al. (2000); the dependence on $\tau$ is characterized by the distribution of the finite time Lyapunov exponent, $\Sigma$ (as defined in Sect. 2.2) for the dominant singular value, $\sigma_{1}(\boldsymbol{x}(t), \tau)$, and by the distribution of the finite sample Lyapunov exponent, $\Lambda$ (as defined in Sect. 2.3) for the local Lyapunov number, $\lambda(\boldsymbol{x}(t), \tau)$. In Fig. 3, the distributions of $\Sigma$ and $\Lambda$ are shown for $\tau=0.01$ (equal to the time step of the nu- merical integrator), $\tau=0.1, \tau=0.5$, and $\tau=1.0$ time unit intervals. For each value of the coupling parameter, the distributions are shown for a single selected initial condition near the separatrix of the pendulum component of the system. (Since the elastic pendulum has no attractor, the results are, strictly speaking, valid only for the trajectory containing the selected initial condition.) As it will be shown later, the neighborhood of the separatrix is an important region of local low predictability. The distributions are strikingly similar for $\tau=0.01$ and $\tau=0.1$ in all panels of Fig. 3, while for the two longer time intervals there are important differences in the distributions. Interestingly, these differences, especially for the finite sample Lyapunov exponent, are much smaller for the two extreme (smallest and largest investigated) values of the coupling parameters. We decided to choose the largest $\tau$ which provides distributions consistent with those for $(\tau=0.01)$, the shortest possible time scale for the discrete maps generated by the numerical solutions. Thus for this paper we consider $\tau=0.1$. As further results of the paper show, $\tau=0.1$ provides good structural resolution and smooth changes of the local predictability measures in the phase space.

The local predictability in the phase space is mapped by Poincaré Sections (e.g. Tabor, 1989; Ott, 1993). Poincaré sections are prepared for a "slow" $\left(\vartheta, p_{\vartheta}\right)$ plane, defined by: $\rho=0, p_{\rho}<0$; and for a "fast" $\left(\rho, p_{\rho}\right)$ plane defined by $\vartheta=0, p_{\vartheta}<0$. The "slow" plane shows the pendulum components of the state vector at instances when the rod is neither stretched nor contracted, and the point mass is moving toward the origin along the radial direction. The "fast" plane, at the same time, shows the spring components of the state vector at instances when the pendulum is crossing through the $\vartheta=0$ line during a clockwise rotation. Each Poincaré section presented in this paper show ten trajectories evolving on the same energy shell defined by $H=1.8$.

In Figs. 4-7, Poincaré sections are shown for four different values of the coupling parameter and the local predictability measures are visualized by colors. Both the local Lyapunov number (panels $a$ and $b$ ) and the dominant singular value (panels $c$ and $d$ ) indicate clear patterns of predictability, though in certain regions of the phase space these patterns can be strikingly different for the two measures.

In what follows, we first focus attention on the slow plane (panels a and c). On this plane, both measures are symmetric about the origin due to the symmetry that the orientation of the angle, $\vartheta$, can be arbitrarily chosen.

When the coupling is weak $(\varepsilon=0.025)$ the Poincaré section is reminiscent of the familiar phase portrait of a pendulum. Since the system is only weakly chaotic in this case and the Lyapunov number is only slightly larger than one, there is a balance between portions of the plane where the local Lyapunov number is greater and smaller than one. The regions of expansion and decay are symmetrically distributed on the Poincaré section: the mirror image of a local Lyapunov number about the $p_{\vartheta}=0$ axis is equal to the inverse of that local Lyapunov number. The largest values of the local Lyapunov number, as can be expected based on the theory, are sim- 

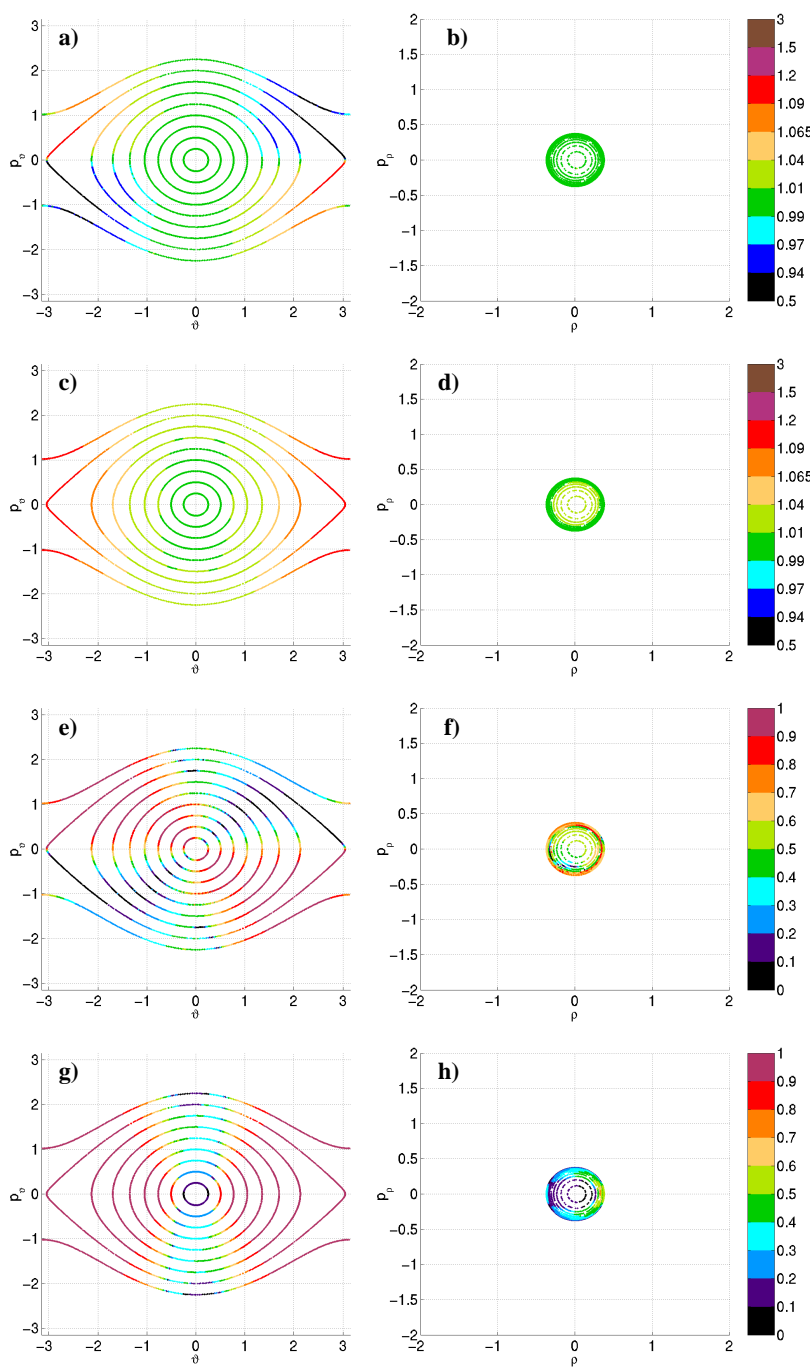

Fig. 4. Left hand side panels show the Poincare section for the "slow" plane $\left(\rho=0, p_{\rho}<0\right)$, while right hand side panels show the Poincare section for the "fast" plane $\left(\vartheta=0, p_{\vartheta}<0\right)$ at a weak coupling $(\varepsilon=0.025)$. Colors represent the local Lyapunov number (panels a and b), the dominant singular value (panels $\mathrm{c}$ and $\mathrm{d}$ ), the projection of the Lyapunov vector on the first right singular vector (panels e and f), and the projection of the first left singular vector on the subsequent first right singular vector (panels $g$ and $h$ ).

ilar to the dominant singular values at the same locations, whereas the smallest values of the local Lyapunov number are similar to the inverse of the associated dominant singular values. These extreme values are located along (and near to) the separatrix of the pendulum, and approaching the origin the values of the predictability measures becomes gradually neutral. The dominant singular values are distributed like the local Lyapunov numbers except for that the mirror image of an expansion about the $p_{\vartheta}=0$ axis is an expansion of the same rate.

As the coupling increases, and the system becomes increasingly chaotic, new local instabilities arise in the region
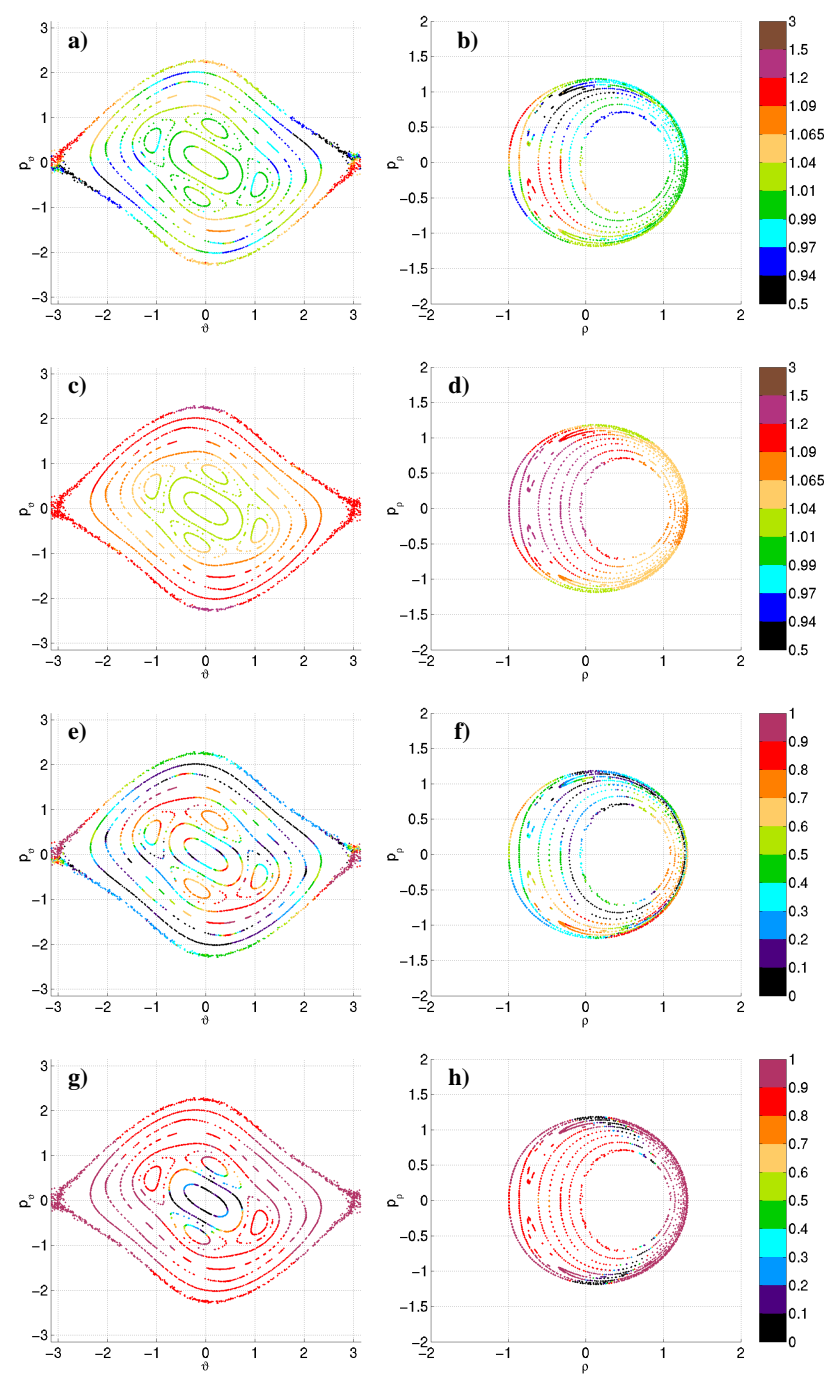

Fig. 5. Same as figure 4, except for $\varepsilon=0.25$.

of the phase space (called region B hereafter), which is associated with small values of the angle and large absolute values of the angular momentum. Both measures agree that the relative importance of this region increases for increasing coupling, but while the dominant singular value tends to emphasize this region, the local Lyapunov number tends to emphasize the region (called region A hereafter) around the unstable subspace of the hyperbolic fixed point of the pendulum.

Another interesting feature is that the local Lyapunov number suggests exceptionally good predictability (decaying Lyapunov vector), even at such large values of the coupling as $\varepsilon=0.4$, for states when the system is moving toward the hyperbolic fixed point of the pendulum. This behavior is further investigated in the next subsection.

When the Poincaré sections for the "slow" plane are plotted using $p_{\rho}>0$ (not shown) instead of $p_{\rho}<0$ the large values of the local Lyapunov number disappear in region $\mathrm{B}$. This indicates that the key to large Lyapunov numbers at 

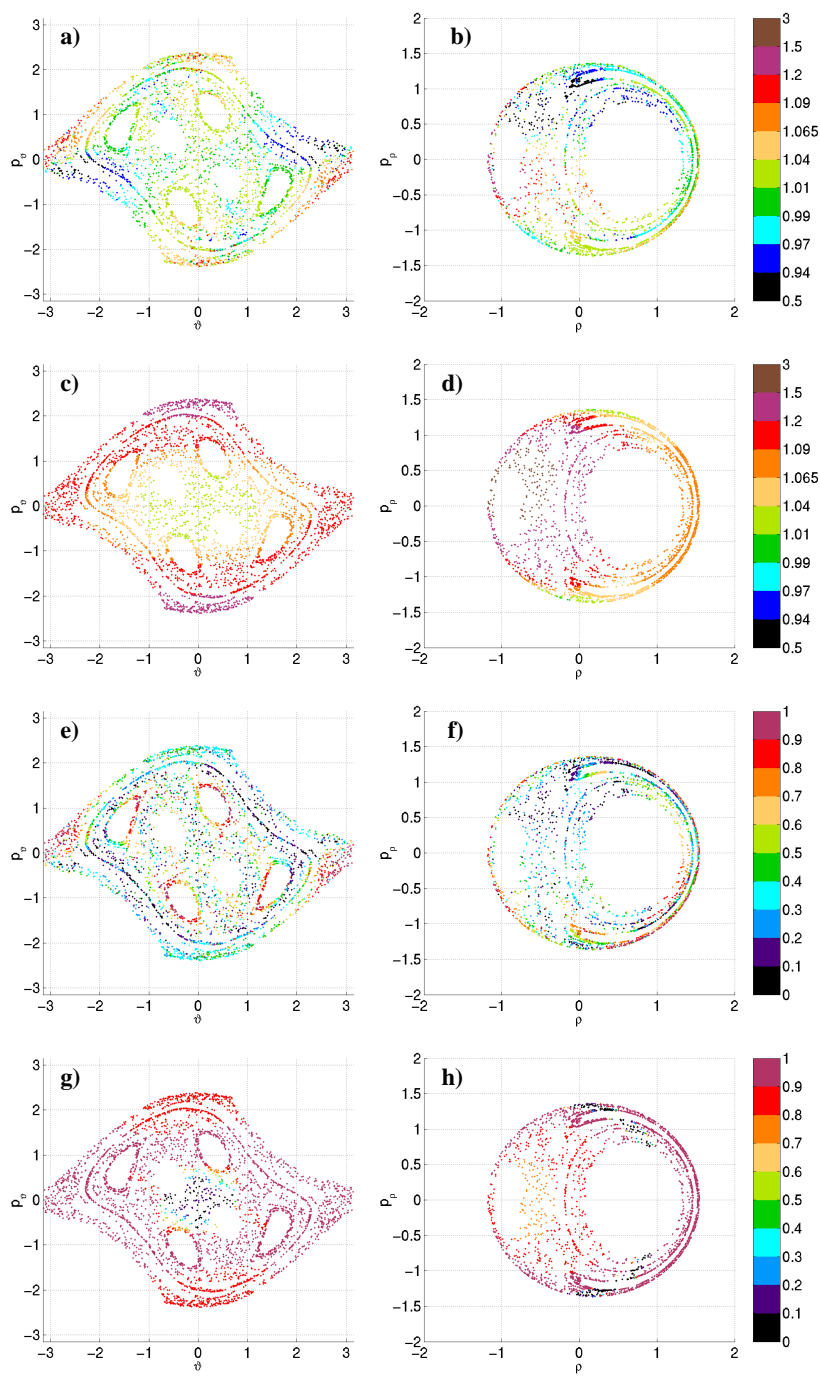

Fig. 6. Same as figure 4, except for $\varepsilon=0.325$.

those locations is the negative radial velocity. This conclusion is well corroborated by the "fast plane" Poincaré sections shown in the right-hand-side panels of Figs. 4-7: The local Lyapunov number, especially for larger values of $\varepsilon$, indicates exceptionally low predictability when the spring is contracted $(\rho<0)$ and moving toward an increasingly contracted state $\left(p_{\rho}<0\right)$, and exceptionally high predictability when the spring is contracted and moving toward a less contracted state. The dominant singular value, on the other hand, suggests that low predictability can occur for an even less contracted spring and regardless the direction of the radial velocity.

\subsection{Which measure should be trusted more?}

The results presented so far raise two important questions: Which measure should be trusted more (i) in the regions where the local Lyapunov number indicates extremely high, and the dominant singular value extremely low predictability
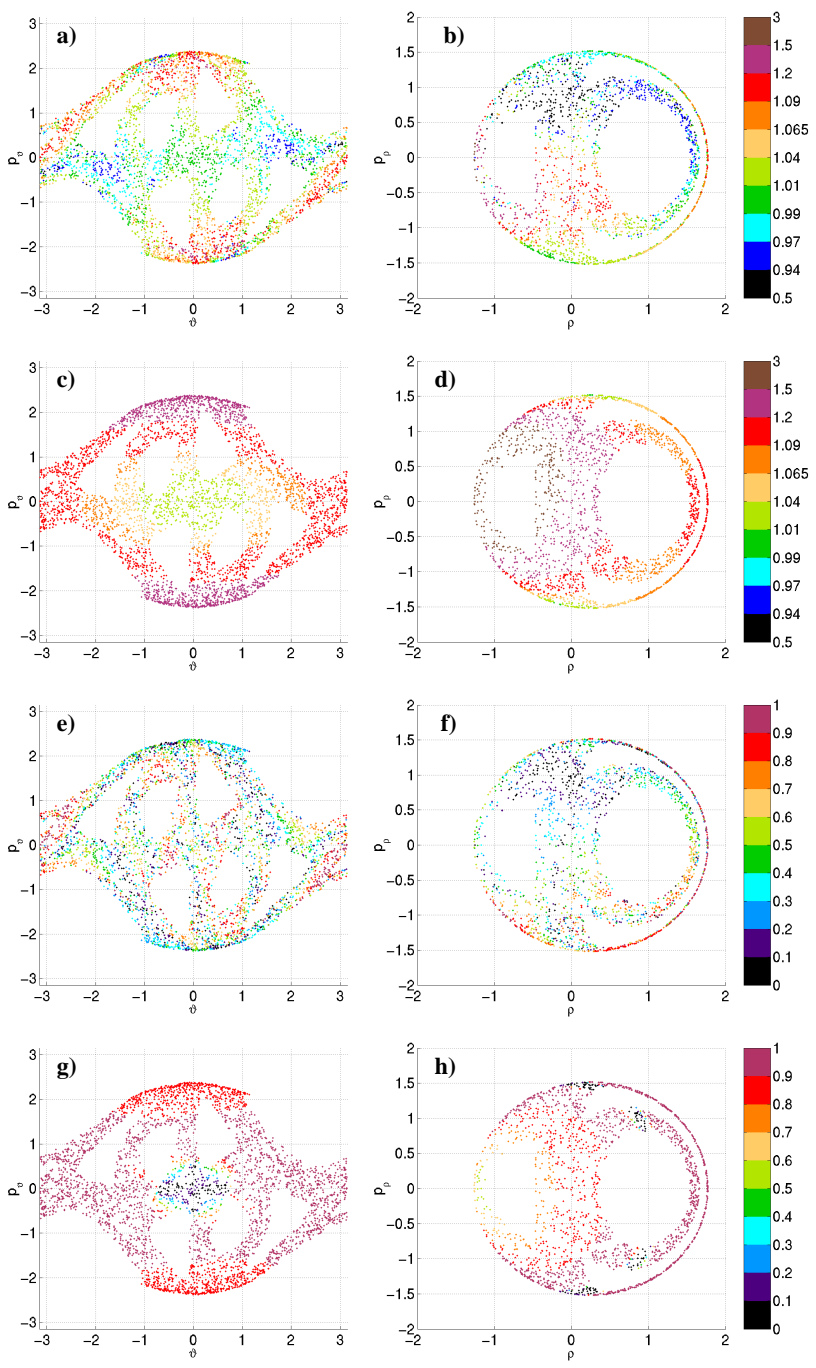

Fig. 7. Same as figure 3, except for $\varepsilon=0.4$.

or (ii) when the two measures indicate different regions as the primary area of low predictability? These questions cannot be answered without exploring the process that makes the Lyapunov and the first singular vectors choose systematically different directions in certain regions of the phase space.

As it was explained in Sect. 2.3, the Lyapunov vector, $\boldsymbol{l}(\boldsymbol{x}(t-\tau))$, can sustain a local expansion rate similar to that of the right singular vector, only if its projection on the first right singular vector, $\boldsymbol{v}(\boldsymbol{x}(t), \tau)$ becomes large. This can happen if and only if (i) the first left and right singular vectors follow each other in a smooth fashion, i.e. if the angle between $\boldsymbol{u}_{1}(\boldsymbol{x}(t), \tau)$ and $\boldsymbol{v}_{1}(\boldsymbol{x}(t+\tau), \tau)$ remains small; and (ii) the first Lyapunov vector has some projection on the first right singular vector at the beginning of the period for which (i) is satisfied.

A comparison of the first and the last four panels of Figs. 4-7 confirms that the two measures of predictability give similar results when the above two conditions are met. 
But the same panels also show that the first Lyapunov vector can become orthogonal to the first right singular vector even if condition (i) is satisfied. This happens primarily at phase space locations where the first Lyapunov vector decays at a rate equal to the inverse of the dominant singular value, indicating that the Lyapunov vector can get "trapped" by a series of smoothly connected, rapidly decaying local directions. To verify this explanation, figures (not shown) similar to panels $(\mathrm{e}-\mathrm{h})$ were also prepared for the fourth, instead of the first, singular vector. As expected, these figures confirmed that at locations where the Lyapunov vector was decaying, the fourth left and right singular vectors followed each other in a smooth fashion and the Lyapunov vector evolved along the direction of the fastest decaying singular vector.

It must be emphasized that the decay of the Lyapunov vector is not associated with isolated phase points, rather it shows sharp patterns on the Poincaré sections. In the related parts of the phase space, uncertainties in the solutions that are associated with initial conditions uncertainties of the distant past, can never be growing. In other words, the Lyapunov vector can sustain significant decay in certain regions of the phase space, even though on average it is growing in a chaotic system.

The large dominant singular values, and the large projection of the first left singular vectors on the following right singular vectors show, at the same time, that uncertainties introduced more recently can grow fast in the same areas where the Lyapunov vector is decaying. These results show that there is no unique best choice for measuring the local predictability in the elastic pendulum, and the proper measure cannot be selected without investigating the primary source of the uncertainty in the initial conditions.

\subsection{Relationship between local predictability and balanced dynamics}

For the elastic pendulum, the analogue to a balanced atmospheric state is a configuration in which the centripetal, the elastic, and the radial components of the gravitational forces are in balance. In this case, the radial acceleration is zero, which means that the last equation of Eq. (8) becomes a nonlinear diagnostic equation between $\vartheta, p_{\vartheta}$, and $\rho$. This algebraic equation is the analogue to the geostrophic balance equation of atmospheric dynamics. This equation allows for the computation of the balanced length of the spring when the "slow" variables, $\vartheta$ and $p_{\vartheta}$, are given. When the state vector is evolved by integrating the full system of Eq. (8), $\rho$ is typically not equal to its hypothetical balanced value, $\rho_{b}$. The difference between $\rho$ and $\rho_{b}$ will be called the unbalanced part of $\rho$ and here it will be determined by computing $\rho_{b}$ from the slow variables first, using the Newton-Raphson method, and then subtracting $\rho_{b}$ from $\rho$.

The right-hand-side panels of Fig. 8 demonstrate that $\rho$ is driven by a combination of balanced motions and high frequency resonances, while the left-hand-side panels of the same figure show the unbalanced part of $\rho$ on the "slow" plane by colors. On these Poincaré sections, the unbalanced
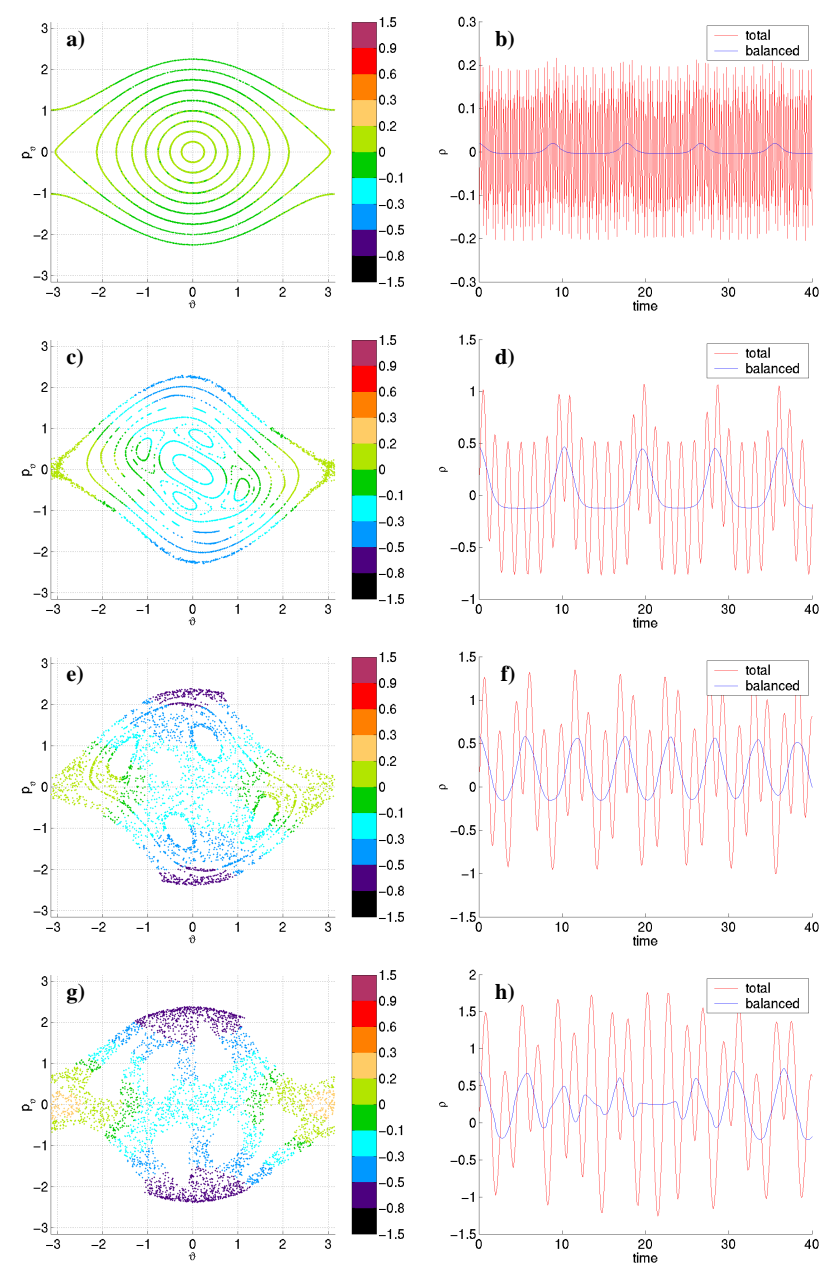

Fig. 8. Left hand side panels show the Poincaré section for the "slow" plane at increasing coupling. The unbalanced component of $\rho$ is shown by colors. Right hand side panels show the time evolution of $\rho$ (solid red line) and its balanced component (solid blue line) for a selected trajectory near the separatrix. The coupling parameters, $\varepsilon$, are 0.025 (panels a and b), 0.25 (panels $\mathrm{c}$ and $\mathrm{d}$ ), 0.325 (panels e and f), and 0.4 (panels g and $h$ ).

component is equal to $-\rho_{b}$, since the "slow" plane is defined by the condition $\rho=0$. The motion is nearly balanced on the slow plane when the coupling is weak $(\varepsilon=0.025)$, but as the coupling increases $\rho$ becomes much smaller than what would be required for the balance, especially in region $\mathrm{B}$. The unbalanced component in region $\mathrm{A}$, on the other hand, is very small regardless the strength of the coupling. Combining these results and those obtained for the local predictability, it can be concluded that the local Lyapunov number identifies a region (region A) as typically least predictable, where the dynamics is nearly balanced. Because in a nearly balanced state the "slow" variables control the dynamics of the system, it is not surprising that the local Lyapunov number found a strong relationship between predictability and the hyperbolic fixed point of the "slow" pendulum component of the system.

To further explore the role unbalanced dynamics play in 

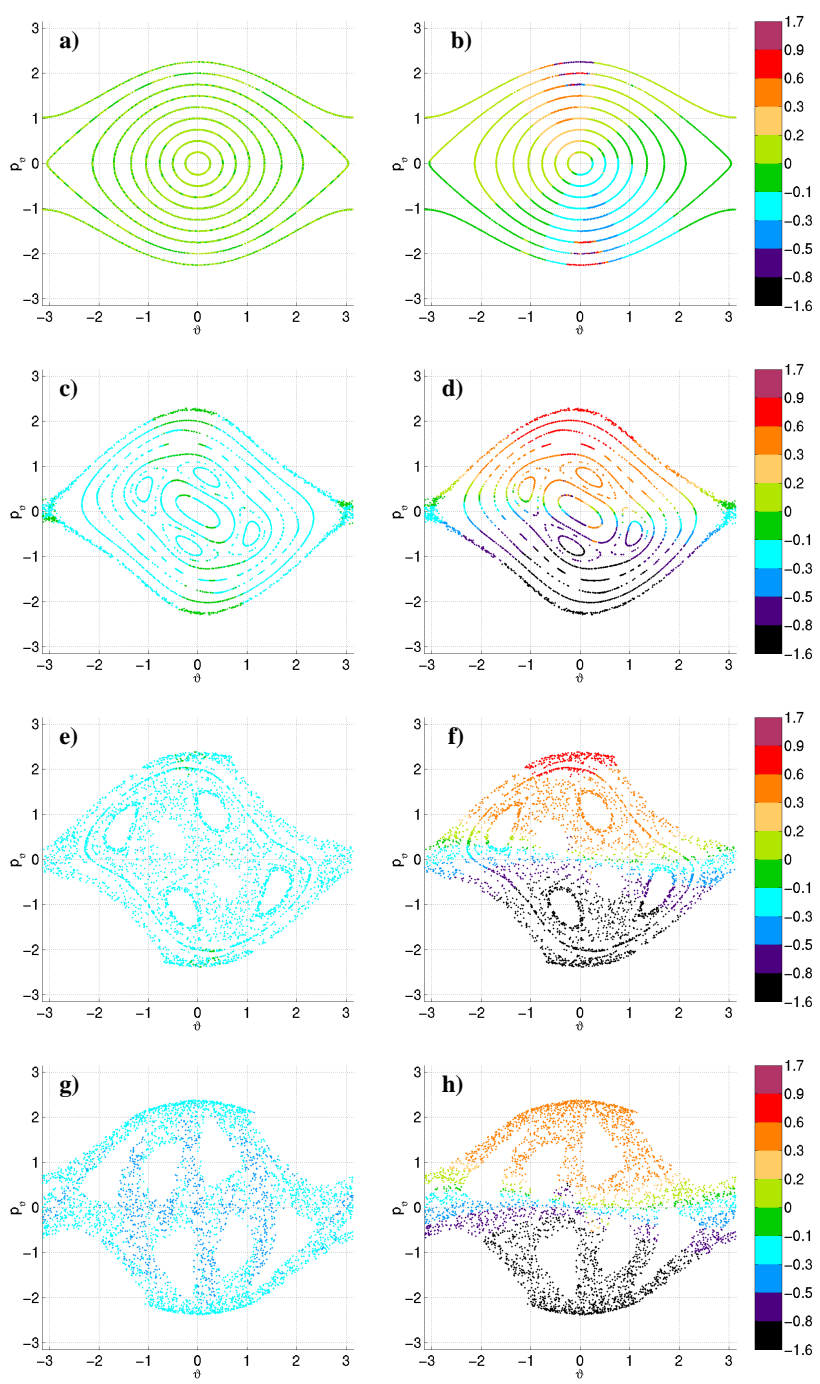

Fig. 9. Left hand side panels show the unbalanced component of $\rho$ for the Lyapunov vector, while the right hand side panels show the same for the first right singular vector. The coupling parameters, $\varepsilon$, are 0.025 (panels a and b), 0.25 (panels $\mathrm{c}$ and d), 0.325 (panels e and f), and 0.4 (panels $g$ and $h$ ).

the local predictability, the unbalanced component is plotted for the normalized Lyapunov and the first right singular vectors (Fig. 9). This figure shows that while the Lyapunov vector (left-hand-side panels) is always nearly balanced on the slow plane independently of the strength of the coupling, the first right singular vector is well balanced only for the very small values of the angular velocity, $p_{\vartheta}$. This shows, on the one hand, that not only the basic state but also the perturbation (Lyapunov and right singular) vectors are well balanced in region $\mathrm{A}$, confirming that the instability in that region is associated with balanced dynamics. On the other hand, the unbalanced nature of the right singular vector plays obvious role in that the dominant singular value emphasizes region $\mathrm{B}$ as the location of exceptionally low predictability.

The results of this section show that the two measures identify different regions of the "slow" plane as the primary location of low predictability, because the Lyapunov vector is always nearly balanced, whereas the right singular vector can be highly unbalanced. It can be concluded, that the relevance of the two measures depends on whether the sources of uncertainties in the solutions are more related to balanced or unbalanced dynamics.

\subsection{Discussion}

Are the results obtained with a simple Hamiltonian model have any relevance regarding atmospheric predictability? To answer this question, it should be recalled first that the adiabatic form of the atmospheric governing equations has a natural Hamiltonian structure (e.g. Shepherd, 1990; Salmon, 1998). Secondly, studies with weakly dissipative atmospheric models found a symmetry between the dominant and the smallest singular values (Reynolds and Palmer, 1998) which was reminiscent of the spectrum of a Hamiltonian system. Though a recent study (Errico et al., 2001) found that diabatic mesoscale processes usually destroy this symmetry, observed synoptic scale instabilities can be reasonably well explained by adiabatic models (e.g. Orlanski and Katzfey, 1991). It can be assumed, therefore, that a Hamiltonian model is a reasonable analogue to synoptic scale atmospheric motions.

Our result on the potential decay of the Lyapunov vector shows that an error pattern generated by an earlier instability has a very specific structure that may even lead to strong decay later. Strong decay is possible only in those regions of the phase space where potentially large growth can occur. This phenomenon is due to the Hamiltonian structure of the system. Although, in contrast to the elastic pendulum, there are more than one expanding phase space directions for the atmosphere, there is growing evidence that synoptic scale atmospheric dynamics can be low dimensional in the regions of a geographically localized instability (Patil et al., 2001). Furthermore, for the dominant atmospheric instabilities, typically there exists a decaying phase space direction in association with each growing direction. For instance, while westward "leaning" perturbation structures lead to baroclinic growth in regions of strong vertical wind shear, eastward leaning structures are decaying at a similar rate. Likewise, in a region of strong horizontal wind shear eddies can either grow or decay through barotropic energy conversion depending on the orientation of their axis.

It can be expected, that in the atmosphere there are complex interactions between the different growing and decaying phase space directions. For instance, it is a well accepted that most synoptic scale waves are generated by baroclinic instabilities of the jet stream and wiped out by barotropic decay in the exit region of the jet (e.g. Whitaker and Dole, 1995). A wave generated by an earlier baroclinic instability can decay because of the orientation of its axis, while another eddy can start growing simply because random noise may project onto a structure in which the axis is pointing into a direction needed for a barotropic growth. In this situation, the local 
Lyapunov number would presumably indicate the decay associated with the decaying wave, while the dominant singular vector could indicate the potential for growing errors.

Our result, that the structure of the first right singular vector is typically highly unbalanced suggests, that in a system for which balanced and the unbalanced motions can be defined, unbalanced perturbations can be expected to lead to rapid instantaneous error growth regardless if an attractor exists or not.

On the practical side, it is essential to explore whether the known sources of uncertainties in the initial conditions can lead to the unbalanced structure required for the rapid growth indicated by the dominant singular value. If not, such choices of the coordinates (inner products) should be preferred so as to efficiently filter the unbalanced features from the dominant singular vectors. The feasibility of finding such coordinates was demonstrated by Ehrendorfer and Errico (1995).

The predictability measures considered in this paper can quantify only that part of the local forecast degradation which is due to sensitivity to the initial conditions. It is important to note, however, that in atmospheric applications, model errors also play an important role. (In our simple model, the only model related error that has some effect on the local forecast accuracy is the local integration error. However, due to the high-accuracy of the integration scheme, this component is negligible compared to the chaos related forecast uncertainties.)

Finally, it should be noted that the magnitude of the uncertainties in the analyses (best available estimates of the true initial state of the atmosphere) is finite and not infinitesimally small as assumed by the predictability measures. The time evolution of the forecast uncertainties, therefore, can rapidly become nonlinear. When this happens, neither the dominant singular value, nor the Lyapunov number can provide correct estimates of predictability. While almost all studies on atmospheric predictability are based on the assumption that the evolution of uncertainties is linear for the first two-three forecast days, the rigorous tests of Gilmour et al. (2001) revealed that the linearity assumption may not even be valid for the entire first forecast day. We are currently in the process of building a three-dimensional variational assimilation system for the elastic pendulum. Our plan is to use this data assimilation system for obtaining estimates of the analysis and forecast uncertainties. Then, these estimates will be used to verify the relevance of the linearity assumption and the capability of the two predictability measures to predict likely forecast errors in the elastic pendulum. The results of these experiments will be presented in a forthcoming paper.

\section{Conclusions}

The two key findings of the study are:

- For the simple model considered, there exist regions where the local Lyapunov number indicates exceptionally high predictability, while the dominant singular value indicates exceptionally low predictability. This can happen, because the Lyapunov vector can become "trapped" by smoothly connected decaying singular vectors, which are orthogonal to the expanding phase space directions. This can lead to an overestimation of predictability when the important forecast errors have sources other than small errors introduced in the distant past.

- The structure of the right singular vector associated with the dominant singular value tends to be significantly more unbalanced than the structure of the Lyapunov vector. Due to this, the local Lyapunov number has a tendency to choose instabilities associated with balanced motion, while the dominant singular value favors instabilities related to highly unbalanced motion. This may lead to an underestimation of predictability when there are no such sources of uncertainty that could create the highly unbalanced structures. Since this result was obtained by a conservative (Hamiltonian) model, it indicates that this phenomenon can occur even if an attractor, as well as off-the-attractor initial states, do not exist.

\section{Appendix A Symmetric composition schemes for the elastic pendulum}

\section{A1 Symmetric composition methods}

The basic existence-uniqueness theorem for ordinary differential equations implies that, for smooth functions $\mathbf{X}(\boldsymbol{x}(t))$, the solution to the initial value problem (1) is defined in some neighborhood of $t=0$ (e.g. Guckenheimer and Holmes, 1983). This solution defines a map between the points $\boldsymbol{x}_{0}$ and $\boldsymbol{x}(t)$ in the n-dimensional phase space. This map, denoted by $\exp (t \mathbf{X})$ (i.e., $\left.\boldsymbol{x}(t)=\exp (t \mathbf{X}) \boldsymbol{x}_{0}\right)$, is called the flow generated by the vector field $\mathbf{X}$ (the argument $\boldsymbol{x}$ is omitted for brevity). Symmetric composition methods can be used to build numerical integrators when it is possible to find a decomposition

$\mathbf{X}=\sum_{i=1}^{k} \mathbf{X}_{i}$

of the vector field $\mathbf{X}$ such that the flows $\exp \left(t \mathbf{X}_{1}\right), \exp \left(t \mathbf{X}_{2}\right)$, .., $\exp \left(t \mathbf{X}_{k}\right)$ all can be determined analytically for a time interval $\Delta t$. Then different order approximations to $\exp (t \mathbf{X})$ can be constructed by the repeated use of the partial flows (Yoshida, 1990). For instance,

$$
\begin{aligned}
\varphi(t) & =\exp \left(t \mathbf{X}_{1}\right) \exp \left(t \mathbf{X}_{2}\right) \ldots \exp \left(t \mathbf{X}_{k}\right)\left(\boldsymbol{x}_{0}\right) \\
& =\boldsymbol{x}(t)+\mathcal{O}\left(t^{2}\right),
\end{aligned}
$$

is a first order integrator, while

$$
\begin{aligned}
S(t) & =\exp \left(\frac{1}{2} t \mathbf{X}_{1}\right) \exp \left(\frac{1}{2} t \mathbf{X}_{2}\right) \ldots \\
& \times \exp \left(t \mathbf{X}_{k}\right) \ldots \exp \left(\frac{1}{2} t \mathbf{X}_{2}\right) \exp \left(\frac{1}{2} t \mathbf{X}_{1}\right)\left(\boldsymbol{x}_{0}\right) \\
& =\boldsymbol{x}(t)+\mathcal{O}\left(t^{3}\right)
\end{aligned}
$$


Table A1. List of integration schemes considered in this paper. Both symmetric (Type $S$ ) and symmetric composed symmetric steps (Type SS) schemes of different orders with varying number of evaluations for each time step $(\mathrm{m})$ are considered

\begin{tabular}{cccc}
\hline Scheme & Order & Type & $\mathrm{m}$ \\
\hline 1 & 1 & $\mathrm{~S}$ & 1 \\
2 & 2 & $\mathrm{~S}$ & 2 \\
3 & 2 & $\mathrm{SS}$ & 2 \\
4 & 4 & $\mathrm{SS}$ & 3 \\
5 & 4 & $\mathrm{SS}$ & 5 \\
6 & 4 & $\mathrm{SS}$ & 5 \\
7 & 4 & $\mathrm{~S}$ & 4 \\
8 & 4 & $\mathrm{~S}$ & 5 \\
9 & 6 & $\mathrm{SS}$ & 7 \\
10 & 6 & $\mathrm{SS}$ & 9 \\
11 & 8 & $\mathrm{SS}$ & 15 \\
12 & 8 & $\mathrm{SS}$ & 17 \\
\hline
\end{tabular}

is a second order integrator.

It is important to notice, that $\varphi(t)$ and $S(t)$ are composite functions. The computation of $\varphi(t)$ for one time step involves the following series of function evaluations: first $\exp \left(t \mathbf{X}_{k}\right)$ is applied to the initial condition $\boldsymbol{x}_{0}$, then $\exp \left(t \mathbf{X}_{k-1}\right)$ is applied to $\exp \left(t \mathbf{X}_{k}\right)\left(\boldsymbol{x}_{0}\right)$, etc. until $\exp \left(t \mathbf{X}_{1}\right)$ is applied to $\exp \left(t \mathbf{X}_{2}\right) \ldots \exp \left(t \mathbf{X}_{k}\right)\left(\boldsymbol{x}_{0}\right)$ in the last step. Once a proper decomposition of the vector field has been obtained, the subscripts $i(i=1, . . k)$ can be assigned in any arbitrary order to the partial vector fields. The integration is carried forward by shifting the origin to $\Delta t$ after each completed time step.

The standard way to construct higher-order schemes out of $S$ is to use a symmetric composed symmetric steps (or type SS) technique of the general form

$S\left(w_{1} t\right) \ldots S\left(w_{(m+1) / 2} t\right) \ldots S\left(w_{1} t\right)$.

The art is to find a set of coefficients $w_{1}, w_{2}, . . w_{n}$ that gives an error term of the required order. Coefficients, not only satisfying this requirement but also minimizing the leading order error term, are available for a variety of different order schemes in McLachlan (1995). This paper also derived optimized coefficients for a number of symmetric (or type $\mathrm{S}$ in short) methods. These schemes use $\varphi^{+}(t)=\varphi(t)$ and $\varphi^{-}(t)=\varphi^{-1}(-t)$ as building blocks to construct schemes of the general form

$\varphi^{+}\left(c_{m} t\right) \varphi^{-}\left(d_{m} t\right) \ldots \varphi^{+}\left(c_{1} t\right) \varphi^{-}\left(d_{1} t\right)$.

The number of evaluations of $\exp \left(t \mathbf{X}_{k}\right)$ per time step is always equal to $m$ for both the S and SS type schemes and if the solution is needed after each time step the number of evaluation per time steps is $m+1$ for the map $\exp \left(t \mathbf{X}_{1}\right)$. For the remaining maps the number of evaluations per time step is $2 m$, which means that a scheme can be further optimized by choosing the computationally most expensive map as $\exp \left(t \mathbf{X}_{k}\right)$ and the computationally least expensive ones as intermediate steps.

\section{A2 Application to the elastic pendulum}

If a partitioning $H=H_{1}+H_{2}+\ldots H_{k}$ of the Hamiltonian exist such that the flows $\exp \left(t \mathbf{J} \nabla H_{1}\right), \exp \left(t \mathbf{J} \nabla H_{2}\right)$, $\ldots, \exp \left(t \mathbf{J} \nabla H_{k}\right)$ can be determined analytically integrators that preserve the symplectic structure and are accurate to any given required order can be constructed by the repeated use of the partial flows (Yoshida, 1990; McLachlan, 1995). In what follows, the above theory is applied to the elastic pendulum equations. The flows $\exp \left(t \mathbf{J} \nabla H_{1}\right), \exp \left(t \mathbf{J} \nabla H_{2}\right)$, .., $\exp \left(t \mathbf{J} \nabla H_{k}\right)$ can be easily determined for a decomposition of the vector field that consists of systems of differential equations with the following two properties (i) in some of the equations the right hand side is zero, i.e the variables on the left hand side of the same equations are constant (ii) the right hand sides of the remaining equations depend only on the constant variables. Such a decomposition of the elastic pendulum equations can be achieved by a 'three-map' splitting $H(\boldsymbol{q}, \boldsymbol{p})=H_{1}(\boldsymbol{q}, \boldsymbol{p})+H_{2}(\boldsymbol{p})+H_{3}(\boldsymbol{q})$, where

$$
\begin{aligned}
H_{1}(\boldsymbol{q}, \boldsymbol{p}) & =\frac{1}{2} p_{1}{ }^{2}\left(1+\varepsilon^{1 / 2} q_{2}\right)^{-2}, \\
H_{2}(\boldsymbol{p}) & =\frac{1}{2} \varepsilon^{-1} p_{2}{ }^{2}, \\
H_{3}(\boldsymbol{q}) & =\frac{1}{2} \varepsilon^{-1} q_{2}{ }^{2}-\left(1+\varepsilon^{1 / 2} q_{2}\right) \cos q_{1},
\end{aligned}
$$

The three differential equations defined by Eq. (A6) are easy to solve and the three elementary maps are

$\exp \left(t \mathbf{J} \nabla H_{1}\right):$

$$
\begin{aligned}
& q_{1}(t)=q_{1}(0)+\left(1+\varepsilon^{1 / 2} q_{2}(0)\right)^{-2} p_{1}(0) t \\
& p_{2}(t)=p_{2}(0)+\varepsilon^{1 / 2}\left(1+\varepsilon^{1 / 2} q_{2}(0)\right)^{-3} p_{1}^{2}(0) t
\end{aligned}
$$

$\exp \left(t \mathbf{J} \nabla H_{2}\right)$ :

$q_{2}(t)=q_{2}(0)+\varepsilon^{-1} p_{2}(0) t$

$\exp \left(t \mathbf{J} \nabla H_{3}\right)$ :

$$
\begin{aligned}
& p_{1}(t)=p_{1}(0)-\left(1+\varepsilon^{1 / 2} q_{2}(0)\right) \sin q_{1}(0) t \\
& p_{2}(t)=p_{2}(0)-\left(\varepsilon^{-1} q_{2}(0)+\varepsilon^{1 / 2} \cos q_{1}(0)\right) t .
\end{aligned}
$$

Steps that do not change the value of the variables (e.g. $\left.q_{1}(t)=q_{1}(0)\right)$ are omitted for brevity and the ordering of the terms $H_{1}, H_{2}$, and $H_{3}$ is not by accident. This particular ordering provides the fastest numerical integrators.

For comparing the efficiency of the different schemes we define work as the number of the evaluations of $\exp \left(t \mathbf{J} \nabla H_{3}\right)$ needed to integrate the model for a unit time interval. This is equal to $m$ times the number of time steps for the unit time interval. This definition was chosen because the evaluation 
of $\exp \left(t \mathbf{J} \nabla H_{3}\right)$ requires two orders of magnitude more CPUtime the computation of the other elementary maps.

We note that the experiments presented in this paper also required the coding of the tangent linear map for all 12 schemes. This was not especially difficult since the tangentlinear operators to the higher order schemes were easily built from the tangent linear operators to the elementary maps (Eq. A7-A9).

Acknowledgements. This paper presents the results of a research supported by the W. M. Keck Foundation. We are especially grateful to D. Devenyi for bringing P. Lynch's thought provoking papers to our attention and for his helpful comments on an earlier version of the manuscript. We would also like to thank E. Kalnay, E. Ott, L. Smith, and two anonymous reviewers for providing helpful comments on an earlier version of the paper.

\section{References}

Anderson, J. L.: Selection of initial conditions for ensemble forecasts in a simple perfect model framework, J. Atmos. Sci., 53, 22-36, 1995.

Arnold, V. I.: Mathematical methods of classical mechanics, Sec. Edition, 508 pp., Springer-Verlag, New York, 1989.

Bokhove, O. and Shepherd, T.: On Hamiltonian balanced dynamics and the slowest invariant manifold, J. Atmos. Sci., 53, 276-297, 1996.

Buizza, R., Tribbia, J., Molteni, F., and Palmer, T.: Computation of optimal unstable structures for a numerical weather prediction model, Tellus, 45A, 388-407, 1993.

Buizza, R. and Palmer, T. N.: The singular vector structure of the of the atmospheric general circulation, J. Atmos. Sci., 52, 14341456, 1995.

Camassa, R.: On the geometry of atmospheric slow manifold, Physica D, 84, 134-139, 1995.

Ehrendorfer, M. and Errico, R. M.: Mesoscale predictability and the spectrum of optimal perturbations, J. Atmos. Sci., 52, 34753500, 1995.

Errico, R. M.: The dynamical balance of leading singular vectors in a primitive equation model, Q. J. Roy. Meteorol. Soc., 126, 1601-1618, 2000.

Errico, R. M., Ehrendorfer, M., and Reader, K.: The spectra of singular values in a regional model, Tellus, 53A, 317-332, 2001.

Farrell, B.: Optimal excitation of neutral Rossby waves, J. Atmos. Sci., 45, 163-172, 1988.

Farrell, B.: Small error dynamics and the predictability of atmospheric flows, J. Atmos. Sci., 47, 2409-2416, 1990.

Frederiksen, J. S.: Singular vectors, finite-time normal modes, and error growth during blocking, J. Atmos. Sci., 57, 312-333, 2000.

Ge, Z. and Marsden, J. E.: Lie-Poisson Hamilton-Jacobi theory and Lie-Poisson integrators, Phys. Lett. A, 133, 134-139, 1988.

Gilmour, I., Smith, L. A., and Buizza, R.: Linear regime duration: Is 24 hours a long time in synoptic weather forecasting? J. Atmos. Sci., 58, 3525-3539, 2001.

Golub, G. H. and Van Loan, C. F.: Matrix computations, The Johns Hopkins University Press, Baltimore, 642, 1983.

Guckenheimer, J. and Holmes, P.: Nonlinear Oscillation, dynamical systems, and bifurcations of vector fields, Springer-Verlag, New York, 459, 1983.
Houtekamer, P. L.: Variation of the predictability in a low order spectral model of the atmospheric circulation, Tellus, 43A, 177190, 1991.

Kádár, B., Szunyogh, I., and Dévényi, D.: On the origin of model errors. Part II. Effects of the spatial discretization for Hamiltonian systems, Időjárás, 102, 71-107, 1998.

Lacarra, J. F. and Talagrand, O.: Short range evolution of small perturbations in a barotropic model, Tellus, 40A, 81-95, 1988.

Legras, B. and Vautard, R.: A guide to Lyapunov vectors, Proceedings of the ECMWF Seminar on Predictability, Reading, England, 143-156, 1996.

Lorenz, E. N.: On the existence of the slow manifold, J. Atmos. Sci., 43, 1547-1557, 1986.

Lorenz, E. N., Predictability: a problem partly solved, Proceedings of the ECMWF Seminar on Predictability, Reading, England, 1$18,1996$.

Lynch, P.: The elastic pendulum: A simple mechanical model of atmospheric balance, Tech. Note 54 of The Irish Meteorological Service, Met Éireann, Dublin, Ireland, 28, 1996.

Lynch, P.: The swinging spring: A simple model of atmospheric balance, in Large-Scale Atmosphere-Ocean dynamics: Vol II: Geometric Methods and Models, Cambridge University Press, 64-108, 2002.

McLachlan, R. I.: On the numerical integration of ordinary differential equations by symmetric composition methods, SIAM J. Sci. Comput., 16, 151-168, 1995.

McLachlan, R. I. and Atela, P.: The accuracy of symplectic integrators, Nonlinearity, 5, 541-562, 1992.

Molteni, F. and Palmer, T. N.: Predictability and finite-time instability of the northern winter circulation, Q. J. Roy. Meteorol. Soc., 119, 73-119, 1993.

Montani A. and Thorpe, A. J.: Mechanisms leading to singularvector growth for FASTEX cyclones, Q. J. Roy. Meteor. Soc., 128, 131-148, 2002.

Orlanski, I. and Katzfey, J.: The life cycle of a cyclone wave in the Southern Hemisphere. Part I: Eddy energy budget, J. Atmos. Sci., 48, 1972-1998, 1991.

Ott, E.: Chaos in dynamical systems, Cambridge University Press, New York, 385, 1993.

Patil, D. J., Hunt, B. R., Kalnay, E., Yorke, J. A., and Ott, E.: Local low dimensionality of atmospheric dynamics, Phys. Rev. Lett., 86, 5878-5881, 2001.

Reynolds, C. A. and Errico, R. M.: Convergence of singular vectors toward Lyapunov vectors, Month. Wea. Rev., 127, 2309-2323, 1999.

Reynolds, C. A. and Palmer, T. N.: Decaying singular vectors and their impact on analysis and forecast correction, J. Atmos. Sci., 55, 3005-3023, 1998.

Salmon, R.: Geophysical fluid dynamics, Oxford University Press, New York, 378, 1998.

Samelson, R. M.: Lyapunov, Floquet, and singular vectors for baroclinic waves, Non. Proc. Geophys., 8, 439-448, 2001.

Sanz-Serna, J. M.: Numerical ordinary differential equations vs. Dynamical systems, The dynamics of numerics and the numerics of dynamics, (Eds) Broomhead, D. S. and Iserles, A., Clarendon Press, Oxford, 81-106, 1992.

Sanz-Serna, J. M. and Calvo, M. P.: Numerical Hamiltonian problems, Chapman and Hall, London, 207, 1994.

Shepherd, T. G.: Symmetries, conservation laws, and Hamiltonian structure in geophysical fluid dynamics, Adv. Geophys., 32, 287338, 1990.

Smith, L. A.: The maintenance of predictability, in Past and present 
variability of the solar-terrestrial system: measurement, data analysis and theoretical models, (Eds) Castagnoli,C. G. and Provenzale, A., Italian Physical Society, IOS Press, Amsterdam, 177-246, 1997.

Smith, L. A., Ziehmann, C., Fraedrich, K.: Uncertainty dynamics and predictability in chaotic systems, Q. J. R. Meteorol. Soc., 125, 2855-2886, 1999.

Szunyogh, I., Kalnay, E., and Toth, Z.: A comparison of Lyapunov and optimal vectors in a low-resolution GCM, Tellus, 49A, 200227, 1997.

Tabor, M.: Chaos and integrability in nonlinear dynamics. An introduction, John Wiley and Sons, New York, 364, 1989.

Talagrand, O.: A study of the dynamics of four-dimensional data assimilation, Tellus, 33, 43-60, 1981.

Thorpe, A. J.: Predictability studies with FASTEX data using the ECMWF IFS, Proceedings of the ECMWF Seminar on Predictability, Reading, England, 185-194, 1996.

Trevisan, A. and Legnani, R.: Transient error growth and local predictability: a study in the Lorenz system, Tellus, 47A, 103-117, 1995.

Trevisan, A. and Pancotti, F.: Periodic orbits, Lyapunov vectors, and singular vectors in the Lorenz system, J. Atmos. Sci., 55, 390-398, 1998.

Trevisan, A., Pancotti, F., and Molteni, F.: Ensemble prediction in a model with flow regimes, Q. J. Roy. Meteorol. Soc., 127, 343358, 2001.

Vannitsem, S. and Nicolis, C.: Lyapunov vectors and error growth patterns in a T21L3 quasi-geostrophic model, J. Atmos. Sci., 54, 347-361, 1997.

Vukicevic, T.: Possibility of skill forecasts based on the finitetime dominant linear solutions for a primitive equation regional model, J. Atmos. Sci., 50, 1777-1791, 1993.

Weintraub, S. H., Differential forms, A complement to vector calculus, Academic Press, San Diego, 256, 1997.

Whitaker, J. S. and Dole, R. M.: Organization of storm tracks in zonally varying flows, J. Atmos. Sci., 52, 1178-1191, 1995.

Yoshida, H.: Construction of higher order symplectic integrators, Phys. Lett. A, 150, 262-268, 1990.

Ziehmann, C., Smith, L. A., and Kurths, J.: Localized Lyapunov exponents and the prediction of predictability, Phys. Lett. A., 271, 237-251, 2000. 